\title{
Prediction of tumour grade and survival outcome using pre-treatment PET- and MRI-derived imaging features in patients with resectable pancreatic ductal adenocarcinoma
}

\author{
Vincent Dunet ${ }^{1} \cdot$ Nermin Halkic $^{2} \cdot$ Christine Sempoux $^{3} \cdot$ Nicolas Demartines $^{2} \cdot$ Michael Montemurro $^{4}$. \\ John O. Prior $^{5}$ (iD) Sabine Schmidt ${ }^{1}$
}

Received: 2 June 2020 / Revised: 29 June 2020 / Accepted: 12 August 2020 / Published online: 26 August 2020

(C) The Author(s) 2020

\begin{abstract}
Objectives To perform a correlation analysis between histopathology and imaging in patients with previously untreated pancreatic ductal adenocarcinoma (PDAC) and to determine the prognostic values of clinical, histological, and imaging parameters regarding overall survival (OS), disease-specific survival (DSS), and progression-free survival (PFS).

Methods This single-centre study prospectively included 61 patients (32 males; median age, 68.0 years [IQR, 63.0-75.0 years]) with histologically confirmed PDAC and following surgical resection who preoperatively underwent ${ }^{18} \mathrm{~F}-\mathrm{FDG}$ PET/CT and DWMRI. On whole lesions, we measured, using a $42 \% \mathrm{SUV}_{\max }$ threshold volume of interest (VOI), the following quantitative parameters: mean and maximum standardised uptake values ( $\mathrm{SUV}_{\text {mean }}$ and $\left.\mathrm{SUV}_{\max }\right)$, total lesion glycolysis (TLG), metabolic tumour volume (MTV), mean and minimum apparent diffusion coefficient $\left(\mathrm{ADC}_{\text {mean }}\right.$ and $\left.\mathrm{ADC}_{\text {min }}\right)$, diffusion total volume (DTV), and MTV/ADC ${ }_{\min }$ ratio. Spearman's correlation analysis was performed to assess relationships between these markers and histopathological findings from surgical specimens (stage; grade; resection quality; and vascular, perineural, and lymphatic invasion). Kaplan-Meier and Cox hazard ratio methods were used to evaluate the impacts of imaging parameters on OS $(n=41)$, DSS $(n=36)$, and PFS $(n=41)$.

Results Inverse correlations between $\mathrm{ADC}_{\text {min }}$ and $\mathrm{SUV}_{\text {max }}(\mathrm{rho}=-0.34 ; p=0.0071)$, and between $\mathrm{SUV}_{\text {mean }}$ and $\mathrm{ADC}_{\text {mean }}$ (rho $=-0.29 ; p=0.026)$ were identified. $\mathrm{ADC}_{\min }$ was inversely correlated with tumour grade (rho $\left.=-0.40 ; p=0.0015\right)$. MTV was an independent predictive factor for OS and DSS, while DTV was an independent predictive factor for PFS.

Conclusion In previously untreated PDAC, ADC and SUV values are correlated. Combining PET-MRI metrics may help predict PDAC grade and patients' survival.
\end{abstract}

Electronic supplementary material The online version of this article (https://doi.org/10.1007/s00330-020-07191-z) contains supplementary material, which is available to authorized users.

John O. Prior

John.Prior@chuv.ch

1 Department of Diagnostic and Interventional Radiology, Lausanne University Hospital and University of Lausanne, Lausanne, Switzerland

2 Department of Visceral Surgery, Lausanne University Hospital and University of Lausanne, Lausanne, Switzerland
3 Institute of Pathology, Lausanne University Hospital and University of Lausanne, Lausanne, Switzerland

4 Department of Oncology, Lausanne University Hospital and University of Lausanne, Lausanne, Switzerland

5 Department of Nuclear Medicine and Molecular Imaging, Lausanne University Hospital and University of Lausanne, Rue du Bugnon 46, CH-1011 Lausanne, Switzerland 


\section{Key Points}

- Minimum apparent diffusion coefficient derived from DW-MRI inversely correlates with tumour grade in pancreatic ductal adenocarcinoma.

- In pancreatic ductal adenocarcinoma, metabolic tumour volume has been confirmed as a predictive factor for patients' overall survival and disease-specific survival.

- Combining PET and MRI metrics may help predict grade and patients' survival in pancreatic ductal adenocarcinoma.

Keywords Carcinoma, Pancreatic ductal · Diffusion magnetic resonance imaging · Fluorodeoxyglucose F-18 · Progression-free survival $\cdot$ Patient outcome assessment

$\begin{array}{ll}\text { Abbreviations } \\ { }^{18} \text { F } & \text { Fluorine-18 } \\ \text { ADC } & \text { Apparent diffusion coefficient } \\ \text { CT } & \text { Computed tomography } \\ \text { DSS } & \text { Disease-specific survival } \\ \text { DTV } & \text { Diffusion tumour volume } \\ \text { DWI } & \text { Diffusion-weighted imaging } \\ \text { FDG } & \text { Fluorodeoxyglucose } \\ \text { IQR } & \text { Interquartile range } \\ \text { MRI } & \text { Magnetic resonance imaging } \\ \text { MTV } & \text { Metabolic tumour volume } \\ \text { OS } & \text { Overall survival } \\ \text { PDAC } & \text { Pancreatic ductal adenocarcinoma } \\ \text { PET } & \text { Positron emission tomography } \\ \text { PFS } & \text { Progression-free survival } \\ \text { PSF } & \text { Point source function } \\ \text { SUV } & \text { Standardised uptake value } \\ \text { TLG } & \text { Total lesion glycolysis } \\ \text { TOF } & \text { Time of flight }\end{array}$

\section{Introduction}

Pancreatic cancer is the second most common digestive cancer and accounts for over 441,000 deaths worldwide [1]. Pancreatic ductal adenocarcinoma (PDAC) is the most frequent pancreatic malignancy and shows a rising incidence [1] with a poor prognosis despite recent advancements in management. Indeed, patients with PDAC have a 5-year survival rate of only $4 \%$ [2]. Notably, pancreatic cancer exhibits a poor response to most chemotherapeutic agents, such that surgery is the only curative treatment.

Accurate preoperative staging is required to select patients who are eligible for surgical resection with negative margins [3]. Magnetic resonance imaging (MRI) can identify patients who are unresectable due to liver metastasis or arterial encasement, while fluorine-18 fluorodeoxyglucose $\left({ }^{18} \mathrm{~F}-\mathrm{FDG}\right)$ positron emission tomography coupled with computed tomography (PET/CT) can identify patients with distant metastatic disease. ${ }^{18}$ F-FDG PET/CT and MRI have proven useful for preoperative differentiation between benign and malignant pancreatic disease [4-7]. Among patients with resectable disease, quantitative imaging metrics can help identifying patients likely to have poor outcomes [8-11], similarly to histological characteristics obtained from surgical specimens [12]. However, there is a paucity of evidence on the correlation between quantitative imaging metrics and pathological features [6-8, 13-15]. Moreover, we wondered if initial ${ }^{18} \mathrm{~F}$ FDG PET/CT and MRI parameters, as well as any clinical and histological parameters, were useful for predicting patient survival in a single population.

The present study aimed to perform a correlation analysis between histopathology and imaging in patients with untreated PDAC and to determine the prognostic value of clinical, histological, and imaging-related parameters in terms of overall survival (OS), disease-specific survival (DSS), and progression-free survival (PFS).

\section{Methods}

\section{Study population}

This study was designed as a transparent reporting of a multivariable prediction model for individual prognosis or diagnosis (TRIPOD) type 1a study to assess the potential benefit of pre-treatment PET- and MRI-derived imaging features in patients with operable PDAC [16]. The TRIPOD checklist is provided in Supplementary Table 1.

From July 2008 to July 2017, all patients with suspected PDAC were prospectively and consecutively enrolled, provided they were not previously treated. The inclusion criteria were age $>18$ years, suspected PDAC without previous treatment, and planned curative surgical resection at our hospital. Exclusion criteria were pregnancy, contraindications to MRI, detection of unresectable tumour on preoperative imaging or during surgery, and non-PDAC lesions on histology. All patients underwent both ${ }^{18}$ F-FDG PET/CT and DW-MRI examination before surgery. Operated patients with confirmed PDAC based on surgical specimens, and with complete imaging and histopathological datasets, were included in a radiopathological correlation analysis and in the follow-up study. All participants gave their written informed consent $48 \mathrm{~h}$ prior to inclusion in the study. The protocol was 
approved by our institutional review board and local research ethics committee (study \#119/08).

\section{Imaging protocols}

The ${ }^{18} \mathrm{~F}$-FDG PET/CT examinations were performed using a Discovery LS scanner until 08/2011 $(n=34)$, and thereafter with a Discovery 690 PET/CT scanner (GE Healthcare; $n=$ 27). Before examination, patients fasted for $>6 \mathrm{~h}$, and blood glucose level was verified to be $\leq 8.3 \mathrm{mmol} / \mathrm{L}$ prior to ${ }^{18} \mathrm{~F}$ FDG administration. Patients were injected with ${ }^{18} \mathrm{~F}$-FDG: $5.5 \mathrm{MBq} / \mathrm{kg}$ until 08/2011, and thereafter with $3.5 \mathrm{MBq} / \mathrm{kg}$ or $309 \pm 81 \mathrm{MBq}$ (range: $158-488 \mathrm{MBq}$ ). At $68 \pm 11 \mathrm{~min}$ (range: 50-95 $\mathrm{min}$ ) after injection, we performed PET acquisition from the vertex to mid-thigh: two-dimensional mode with 6-7 steps of 3-5 min for the Discovery LS or threedimensional mode with $8-9$ steps of 2 min for the Discovery 690 (mean duration: $20 \pm 5 \mathrm{~min}$; range: $16-35 \mathrm{~min}$ ). All PET acquisition and reconstruction parameters are displayed in Supplementary Table 2. For attenuation correction, the PET acquisition followed an unenhanced MDCT acquisition from the vertex to mid-thigh: $140 \mathrm{kV}, 80 \mathrm{~mA}$, pitch $1.5,0.5 \mathrm{~s} /$ rotation, 5-mm slice thickness until 08/2011; and $120 \mathrm{keV}, 80$ 200 AutomA/SmartmA, pitch 1.375, and 3.3-mm slice thickness thereafter. Standardised uptake values (SUV) were corrected for body mass [17, 18]. Between scanners, stability of PET data of our centre was ensured by biannually acquiring a uniform phantom over the study period [19].

MR data were acquired using a 1.5-T scanner $(n=33$, Symphony or Aera; Siemens Healthcare) or a 3.0-T scanner ( $n=28$; Skyra, Prisma, or Verio, Siemens Healthcare) with an 18-channel phased-array body coil covering the upper abdomen, combined with a 32-channel spine coil. MR acquisition was performed as described in Supplementary Table 3 and included a transverse single-shot spin-echo echo-planar DWI sequence in three orthogonal directions (frequency-encoding, phase-encoding, and slice selection) with three $b$-values ranging from 50 to $800 \mathrm{~s} / \mathrm{mm}^{2}$ in increasing order. Pixel-to-pixel ADC maps were generated from DW-MRI sequences using Siemens software.

\section{Imaging analysis}

${ }^{18}$ F-FDG PET and DW-MR data were analysed by one reader having 9 years of experience in abdominal imaging (nuclear medicine and radiology) on the same workstation (Advantage Workstation 4.6, GE Healthcare). Quantitative parameter values were measured over the pancreatic lesions. To assess the whole tumour, quantitative parameters using volume of interest (VOI), embedding the entire pancreatic lesions, were evaluated.

On unenhanced ${ }^{18} \mathrm{~F}$-FDG PET/CT images, a $42 \% \mathrm{SUV}_{\max }$ threshold VOI around each visible pancreatic lesion was drawn $[20,21]$. On a per-lesion basis, we recorded the $\mathrm{SUV}_{\text {max }}, \mathrm{SUV}_{\text {mean }}$, metabolic tumour volume (MTV) in millilitres, and total lesion glycolysis $\left(\mathrm{TLG}=\mathrm{SUV}_{\text {mean }} \times\right.$ MTV).

On DW-MR images, we manually drew a VOI encompassing the whole lesion on the intermediate of the three $b$-values. We then copied and pasted this VOI on the ADC maps to measure the corresponding mean and minimum $\mathrm{ADCs}\left(\mathrm{ADC}_{\text {mean }}\right.$ and $\left.\mathrm{ADC}_{\text {min }}\right)$. Compared with the twodimensional ROI method, the VOI method was preferred because it demonstrated good inter-observer reproducibility for both $\mathrm{ADC}_{\text {mean }}$ and $\mathrm{ADC}_{\text {min }}$ while containing the areas of the tumour with the highest cellular density in the tumour [22]. The diffusion tumour volume (DTV) was defined as the VOI volume in millilitres. Finally, we calculated the MTV/ADC ${ }_{\min }$ ratio [10].

\section{Histopathological analysis}

All surgical specimens were consecutively subjected to macroscopical and microscopical analyses by a boardcertified pathologist who specialised in digestive oncology with a practical experience of 20 years. An internal standardised protocol was used to assess pTNM, grade (1, well-differentiated; 2 , moderately differentiated; 3 , poorly differentiated; and 4, undifferentiated), resection quality (R0 or $\mathrm{R} 1$ ), vascular invasion (V score of 0 if absent, and 1 if present), perineural invasion ( $\mathrm{Pn}$ score of 0 if absent, or 1 if present), and lymphatic invasion (L score of 0 if absent, or 1 if present). Over the study time period, pTNM staging changed; thus, the pathology reports were reviewed and adapted according to the most recent UICC classification [23].

\section{Follow-up and outcome}

Patients with PDAC verified from surgical specimens were included in the follow-up part. OS was defined as the time from surgery until death from any cause. DSS was defined as the time from surgery until PDAC-related death (with death not related to PDAC being censored). PFS was defined as the time from surgery until progression of the oncological disease (with death not related to PDAC and arising before progression being censored). Follow-up data as of July 2018 were collected from patients' medical history available in the hospital information system, and using a questionnaire sent to the patients' referring physicians.

\section{Statistical analysis}

All statistical analyses were performed using Stata 15.1 software (StataCorp). Continuous variables are presented as median [interquartile range (IQR)] and categorical variables as number and/or percentage (\%) and were compared using the 
Wilcoxon rank-sum test and the Fisher's exact test, respectively. For the radiopathological correlation analysis $(n=61$ patients), we calculated the Spearman's correlation coefficient to assess the relationships between quantitative parameter values $\left(\mathrm{SUV}_{\text {max }}, \mathrm{SUV}_{\text {mean }}, \mathrm{MTV}, \mathrm{TLG}, \mathrm{ADC}_{\text {min }}, \mathrm{ADC}_{\text {mean }}, \mathrm{DTV}\right.$, and $\mathrm{MTV} / \mathrm{ADC}_{\min }$ ratio) and histopathological findings (stage; grade; resection; and vascular, perineural, and lymphatic invasion). Imaging metrics were compared between patients scanned with different PET and MRI devices to evaluate potential bias.

For survival analysis ( $n=41$ patients), each continuous variable was dichotomised using optimal cutoff values determined by the Youden method on receiver operating characteristic (ROC) curves. Outcome was analysed using the Kaplan-Meier method with event-free survival curves and compared using the log-rank test. Univariate and stepwise multivariate analyses were performed to identify independent predictors of death (OS and DSS) and progression (PFS) using the Cox proportional hazard ratio model with the clinical parameters (sex, stage), imaging parameters, magnetic resonance strength field, and PET type. Stepwise multivariate analysis was performed taking into account variables with a $p<0.10$ for forward and $p<0.05$ for backward selection. A $p$ value of $<0.05$ was considered to indicate statistical significance. Bonferroni-corrected $p$ values were calculated for multivariate analysis and are noted in table footnotes.

\section{Results}

\section{Study population's characteristics}

Of the initially enrolled 87 patients, 6 were excluded from radiopathological correlation analysis because they were
Fig. 1 Study flowchart showing inclusion and exclusion criteria

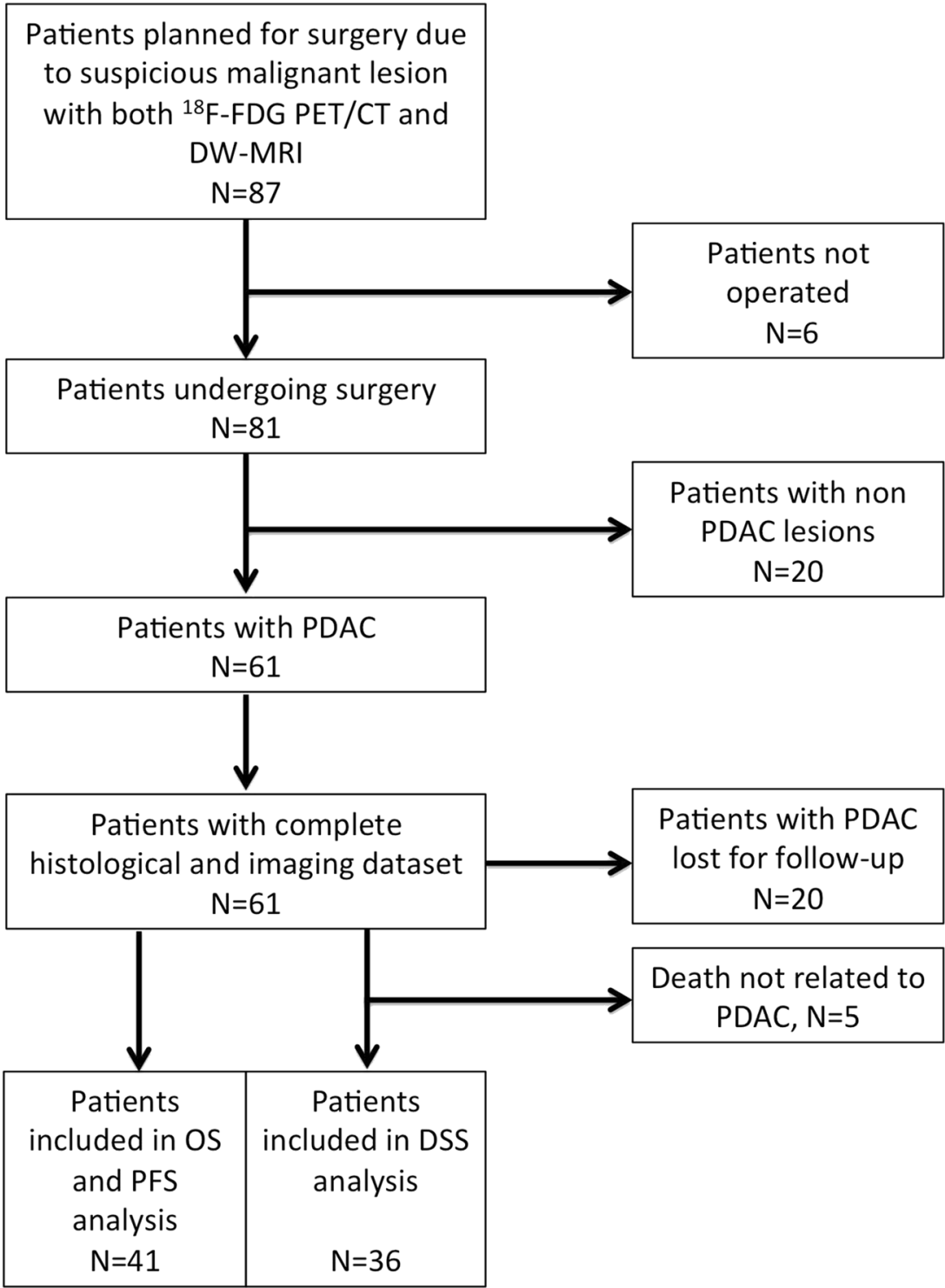


ultimately considered unresectable, and 20 were excluded because they had non-PDAC lesions. The radiopathological correlation analysis included 61 patients ( 32 males; mean age, 68 years [IQR, 63-75 years]).

All 61 included patients underwent both ${ }^{18}$ F-FDG PET/CT and DW-MR examinations within 3 days [IQR, 1-7 days], followed by surgical resection within 10 days [IQR, 621 days]. Of these patients, 48 underwent cephalic duodenopancreatectomy, 2 total pancreatectomy, and 11 distal pancreatectomy. Additionally, 52 patients underwent adjuvant chemotherapy with gemcitabine $(n=46)$, gemcitabinecapecitabine $(n=3)$, FOLFIRINOX $(n=2)$, or Xeloda $(n=1)$.

Twenty patients were lost for follow-up evaluation, and five patients died from non-PDAC-related causes and were excluded from the DSS analysis (Fig. 1). Thus, the survival analysis included a total of 41 patients (21 males; median age, 68 years [IQR, 61-75 years]) (Table 1 ).

\section{Patients characteristics, tumour parameters, and correlation between histopathology and imaging}

Quantitative parameters were similar between patients scanned on different PET scanners and at different MRI strength field (all $p \geq 0.15$, Supplementary Table 4). We identified inverse correlations between $\mathrm{SUV}_{\max }$ and $\mathrm{ADC}_{\min }$ (rho $=-0.34, p=0.0071$ ) and between $\mathrm{SUV}_{\text {mean }}$ and $\mathrm{ADC}_{\text {mean }}(\mathrm{rho}=-0.29, p=0.0026)$ (Figs. 2 and 3). $\mathrm{ADC}_{\text {min }}$ was inversely correlated with TLG (rho $=-0.40, p=0.0015)$ and MTV (rho $=-0.28, p=0.032$ ), while $\mathrm{ADC}_{\text {mean }}$ was only significantly correlated with TLG (rho $=-0.30, p=0.017$ ). We detected a positive correlation between MTV and DTV (rho $=0.77, p<0.0001$ ). Only $\mathrm{ADC}_{\min }$ values were inversely correlated with the tumour grade (rho $=-0.40, p=0.0015$ ) (Table 2).

\section{Survival analysis}

The median follow-up duration was 1.7 years (IQR: 0.8-2.3, range: $0.1-7.3$ years) for the 41 patients included in the OS and PFS analyses and 1.7 years (IQR: 0.8-2.4 years, range: $0.3-7.3$ years) for the 36 patients included in the DSS analysis. Only MTV had significant independent prognostic value for OS (Table 3) and for DSS (Table 4). DTV was an independent predictive factor for PFS (Table 5). OS, PFS, and DSS were not associated with the type of PET scanner or magnetic resonance strength field used (Tables 3,4 , and 5, all $p \geq 0.14$ ).

\section{Discussion}

Our study yielded three main results. First, an inverse correlation between $\mathrm{ADC}$ and SUV was found, while only $\mathrm{ADC}_{\min }$ was significantly correlated with tumour grade in PDAC
Table 1 Patients' and tumours' characteristics $(n=61)$

\begin{tabular}{|c|c|}
\hline Variables & PDAC, $N=61$ \\
\hline Age (years) & $68.0[63.0-75.0]$ \\
\hline Sex, male/female & $32 / 29$ \\
\hline Tumour stage, $1 \mathrm{a} / 1 \mathrm{~b} / 2 \mathrm{a} / 2 \mathrm{~b} / 3 / 4$ & $1 / 3 / 4 / 29 / 21 / 3$ \\
\hline Tumour grade, $1 / 2 / 3$ & $9 / 36 / 16$ \\
\hline Resection, $0 / 1$ & $30 / 31$ \\
\hline Perineural invasion, $0 / 1$ & $2 / 59$ \\
\hline Vascular invasion, $0 / 1$ & $12 / 49$ \\
\hline Lymphatic invasion, $0 / 1$ & $14 / 47$ \\
\hline $\mathrm{SUV}_{\max }(\mathrm{g} / \mathrm{mL})$ & $7.6[5.7-8.9]$ \\
\hline $\operatorname{SUV}_{\text {mean }}(\mathrm{g} / \mathrm{mL})$ & $4.2[3.1-5.2]$ \\
\hline $\operatorname{MTV}\left(\mathrm{cm}^{3}\right)$ & $9.4[6.0-15.6]$ \\
\hline TLG $\left(\mathrm{g} \cdot \mathrm{cm}^{3} / \mathrm{mL}\right)$ & $44.7[21.5-67.3]$ \\
\hline $\mathrm{ADC}_{\min }\left(10^{-6} \mathrm{~mm}^{2} / \mathrm{s}\right)$ & $718[600-950]$ \\
\hline $\mathrm{ADC}_{\text {mean }}\left(10^{-6} \mathrm{~mm}^{2} / \mathrm{s}\right)$ & $1430[1258-1630]$ \\
\hline DTV $\left(\mathrm{cm}^{3}\right)$ & $8.4[5.8-14.9]$ \\
\hline MTV/ADC ${ }_{\min }$ ratio $\left(\times 10^{3}\right)$ & $14.9[7.2-22.8]$ \\
\hline
\end{tabular}

$A D C$, apparent diffusion coefficient; $D T V$, diffusion tumour volume; $M T V$, metabolic tumour volume; $P D A C$, pancreatic ductal adenocarcinoma; $S U V$, standardised uptake value; $T L G$, total lesion glycolysis. The right column indicates numbers or median [interquartile range]

patients. Second, MTV was a significant independent predictive factor for OS and DSS. Third, we demonstrated that DTV was an independent predictive factor for PFS. Overall, these results suggest that the combination of multiple PET-MRI metrics may help in the evaluation of tumour grade and prediction of PDAC patients' survival.

Several prior studies have included radiopathological correlation analyses in patients with PDAC undergoing DW-MRI or ${ }^{18} \mathrm{~F}$-FDG PET/CT, but few have included patients who

Table 2 Correlation between imaging and pathological parameters in PDAC

\begin{tabular}{lrrrrr}
\hline & \multicolumn{1}{l}{$\mathrm{G}$} & \multicolumn{2}{l}{$\mathrm{R}$} & \multicolumn{1}{l}{ Pn } & \multicolumn{1}{l}{$\mathrm{L}$} \\
\hline SUV $_{\text {max }}$ & 0.20 & -0.13 & 0.04 & -0.24 & 0.01 \\
SUV $_{\text {mean }}$ & 0.25 & -0.12 & 0.05 & -0.24 & 0.02 \\
MTV & -0.02 & 0.15 & 0.15 & -0.02 & -0.03 \\
TLG & 0.07 & 0.04 & 0.14 & -0.07 & 0.01 \\
ADC $_{\text {min }}$ & $-0.40 *$ & 0.05 & 0.06 & 0.09 & 0.05 \\
ADC $_{\text {mean }}$ & -0.24 & 0.07 & -0.05 & -0.02 & -0.04 \\
DTV $_{\text {MTV/ADC }}$ & -0.03 & 0.26 & 0.05 & -0.13 & -0.13 \\
\hline
\end{tabular}

Values shown are Spearman's rho values: $* p=0.0015, p>0.05$ for all other items. $A D C$, apparent diffusion coefficient; $D T V$, diffusion tumour volume; $G$, grade; $L$, lymphatic invasion; $M T V$, metabolic tumour volume; $P D A C$, pancreatic ductal adenocarcinoma; $P n$, perineural invasion; $R$, quality of resection; $S U V$, standardised uptake value; $T L G$, total lesion glycolysis; $V$, vascular invasion 
Fig. 2 Correlation analysis between standardised uptake values (SUV in $\mathrm{g} / \mathrm{mL}$ ) and apparent diffusion coefficient (ADC in $10^{-6} \mathrm{~mm}^{2} / \mathrm{s}$ ) values

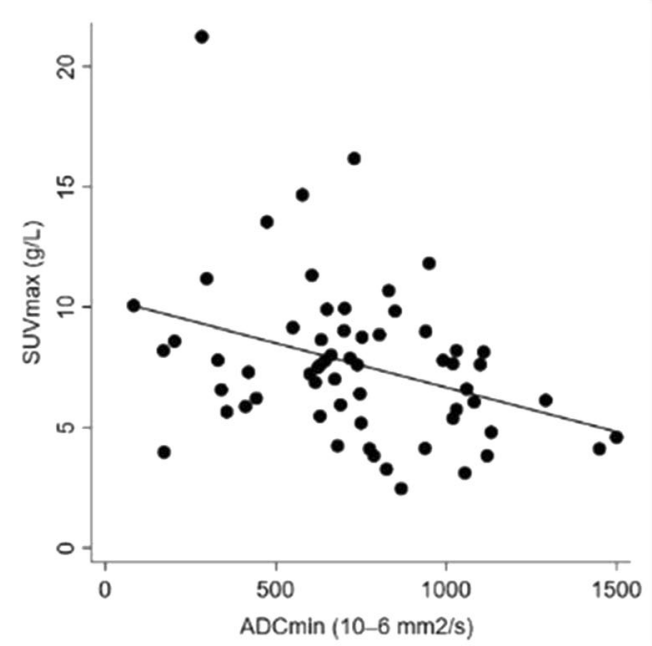

underwent both examinations prior to surgery. To our knowledge, this is the first study to report a significant inverse correlation between $\mathrm{ADC}_{\text {min }}$ and tumour grade in PDAC. No significant correlation between $\mathrm{ADC}_{\text {mean }}$ or ${ }^{18} \mathrm{~F}$-FDG PETderived metrics and pathological parameters was observed.

Prior studies have reported discordant results regarding the relationship between $\mathrm{ADC}_{\text {min }}$ or $\mathrm{ADC}_{\text {mean }}$ and pathological findings. Chen et al [10] found a non-significant inverse association between $\mathrm{ADC}_{\min }$ and tumour differentiation, and Kurosawa et al [24] reported that lower $\mathrm{ADC}_{\text {mean }}$ was associated with lower tumour differentiation. However, other authors [15, 25] have not described any significant association between $\mathrm{ADC}_{\text {mean }}$ and tumour grade. By nature, PDAC are fibrotic tumours; thus, some authors suggest that varying ADC values may be related to differences in fibrosis [26]. Nevertheless, this possibility is not supported by two recent studies [6, 15]. Furthermore, no correlation has been identified between $\mathrm{ADC}_{\text {mean }}$ and tumour microvessel or cell density [15]. Hayano et al [7] reported that tumours with low $\mathrm{ADC}_{\text {min }}$ exhibited a deeper invasion into the portal venous system and extrapancreatic nerve plexus compared with tumours with high $\mathrm{ADC}_{\text {min. }}$. These conflicting results may be related to the heterogeneity of study populations, the variations in evaluated pathological parameters, and/or the different methods used for ADC measurement. Indeed, different authors have used single-ROI, multiple-ROI, or VOI, which can influence the reproducibility of ADC values [22]. Overall, the exact meaning of ADC values remains unclear and is likely related to an averaged multifactorial effect of tumour grade, cell density, and microenvironment.

Discordant results have also been published regarding the correlation between ${ }^{18} \mathrm{~F}$-FDG uptake and proliferative activity. In a study including all histological subtypes of pancreatic cancers, $\mathrm{Hu}$ et al [4] reported a positive correlation between $\mathrm{SUV}_{\text {max }}$ and Ki-67, while Buck et al [14] found no statistical correlation. Ahn et al [13] detected a significant association between $\mathrm{SUV}_{\text {mean }}$ and tumour grade, while Im et al [8] reported no significant association between ${ }^{18} \mathrm{~F}$-FDG parameters and tumour grade or perineural invasion, which agrees with the present results. However, Im et al also noted that TLG and MTV were marginally associated with lymphovascular invasion. Overall, these contradictory results suggest a crucial need for international standardisation of ADC measurements, and for large, multicentric studies, assessing the relationship between ${ }^{18}$ F-FDG PET- and DWI-derived parameters and pathological findings in resectable PDAC.

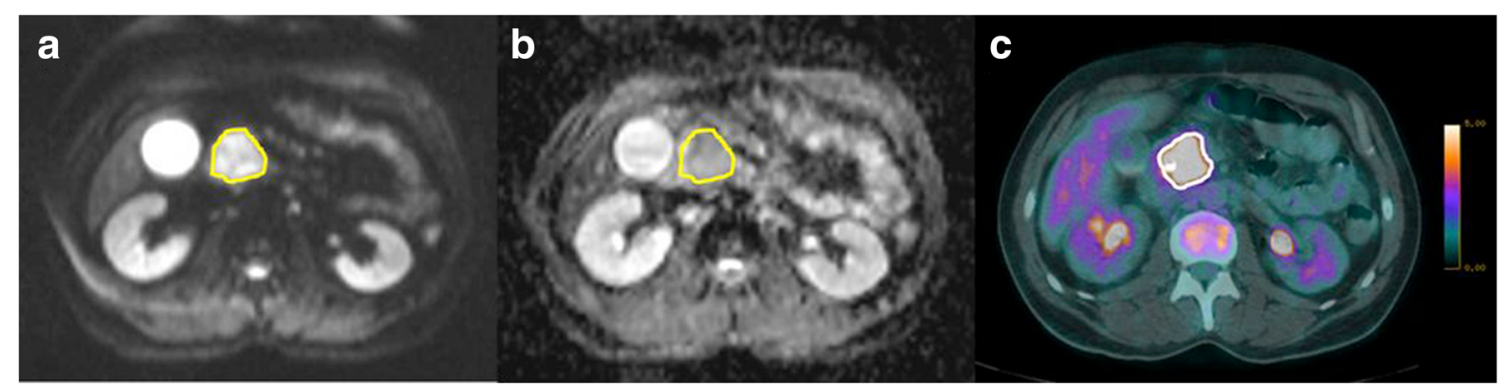

Fig. 3 Example of a patient with untreated PDAC. Diffusion-weighted MRI (a, $b$-value: $800 \mathrm{~s} / \mathrm{mm}^{2}$ ), ADC map (b), and PET/CT (c) were acquired in a 54-year-old male who underwent cephalic duodenopancreatectomy for a pT2N2M0 pancreatic ductal adenocarcinoma. The $\mathrm{ADC}_{\text {mean }}, \mathrm{SUV}_{\text {mean }}, \mathrm{MTV}$, and $\mathrm{MTV} / \mathrm{ADC}_{\text {min }}$ ratio were $1855 \times 10^{-6} \mathrm{~mm}^{2} / \mathrm{s}, 5.2 \mathrm{~g} / \mathrm{mL}, 20 \mathrm{~mL}$, and $21 \times 10^{-3}$, respectively 
Table 3 Predictive factors of OS $(n=41)$

\begin{tabular}{|c|c|c|c|c|c|c|}
\hline \multirow[t]{2}{*}{ Variables } & \multicolumn{3}{|c|}{ Univariate analysis } & \multicolumn{3}{|c|}{ Stepwise multivariate analysis } \\
\hline & HR & $95 \% \mathrm{CI}$ & $p$ value & HR & $95 \% \mathrm{CI}$ & $p$ value \\
\hline Sex & 0.87 & $0.46-1.62$ & 0.65 & - & - & - \\
\hline 1.5- vs. 3.0-T MRI & 1.0 & $0.66-1.52$ & 0.99 & - & - & - \\
\hline Discovery LS vs. 690 & 0.85 & $0.45-1.62$ & 0.63 & - & - & - \\
\hline Stage $1-2$ vs. $3-4$ & 1.46 & $0.76-2.80$ & 0.25 & - & - & - \\
\hline $\mathrm{SUV}_{\max }>7.3(\mathrm{~g} / \mathrm{mL})$ & 1.49 & $0.77-2.86$ & 0.24 & - & - & - \\
\hline $\mathrm{SUV}_{\text {mean }}>4.5(\mathrm{~g} / \mathrm{mL})$ & 1.22 & $0.65-2.33$ & 0.53 & - & - & - \\
\hline MTV > $6.33\left(\mathrm{~cm}^{3}\right)$ & 2.31 & $1.07-5.02$ & 0.034 & 10.9 & $2.09-56.9$ & 0.005 \\
\hline TLG $>24.77\left(\mathrm{~g} \cdot \mathrm{cm}^{3} / \mathrm{mL}\right)$ & 1.80 & $0.87-3.71$ & 0.11 & 0.20 & $0.05-0.93$ & 0.040 \\
\hline $\mathrm{ADC}_{\min }>939\left(10^{-6} \mathrm{~mm}^{2} / \mathrm{s}\right)$ & 0.79 & $0.39-1.60$ & 0.51 & - & - & - \\
\hline $\mathrm{ADC}_{\text {mean }}>1282\left(10^{-6} \mathrm{~mm}^{2} / \mathrm{s}\right)$ & 0.59 & $0.29-1.21$ & 0.15 & - & - & - \\
\hline DTV $>4.72\left(\mathrm{~cm}^{3}\right)$ & 2.27 & $0.98-5.26$ & 0.056 & - & - & - \\
\hline $\mathrm{MTV} / \mathrm{ADC}_{\min }$ ratio $>8.31\left(10^{3}\right)$ & 1.94 & $0.95-3.98$ & 0.071 & - & - & - \\
\hline
\end{tabular}

$A D C$, apparent diffusion coefficient; $D T V$, diffusion tumour volume; $M T V$, metabolic tumour volume; $O S$, overall survival; $P D A C$, pancreatic ductal adenocarcinoma; $S U V$, standardised uptake value; $T L G$, total lesion glycolysis. Bonferroni-corrected $p$ value was 0.009 for MTV and 0.080 for TLG
Several studies have demonstrated a significant correlation between $\mathrm{ADC}$ and SUV values in gastrointestinal tumours $[18,27-30]$. However, few such studies have been performed in pancreatic cancer $[10,31]$. In agreement with these results $[10,31]$, our present study showed significant inverse correlations between $\mathrm{ADC}_{\text {min }}$ and $\mathrm{SUV}_{\text {max }}$, and between $\mathrm{ADC}_{\text {mean }}$ and $\mathrm{SUV}_{\text {mean }}$ in patients with PDAC. Like Chen et al [10], we also detected a significant correlation between TLG and $\mathrm{ADC}_{\text {min }}$.

To date, few studies have examined the prognostic value of multiparametric PET-MRI. It remains unclear how PET and DWI parameters could be combined for use in the prognostic stratification of PDAC patients [26]. In a small sample of 17 patients operated for pancreatic ductal carcinoma, Chen et al [32] found better OS in patients with tumours exhibiting high $\mathrm{ADC}_{\text {min }}$ values compared to those with low $\mathrm{ADC}_{\text {min }}$ values. Similarly, Kurosawa et al [24] reported better OS in patients with tumours presenting high $\mathrm{ADC}_{\text {mean }}$ values than those with low $\mathrm{ADC}_{\text {mean }}$ values. Garces-Descovich et al [33] demonstrated that lower ADC values in PDAC were associated with worse 4-year OS. However, in agreement with Sakane et al [31], our findings showed no significant prognostic value for $\mathrm{ADC}_{\text {mean }}$ or $\mathrm{ADC}_{\text {min }}$.
Table 4 Predictive factors of DSS $(n=36)$

\begin{tabular}{|c|c|c|c|c|c|c|}
\hline \multirow[t]{2}{*}{ Variables } & \multicolumn{3}{|c|}{ Univariate analysis } & \multicolumn{3}{|c|}{ Stepwise multivariate analysis } \\
\hline & HR & $95 \% \mathrm{CI}$ & $p$ value & HR & $95 \% \mathrm{CI}$ & $p$ value \\
\hline Sex & 0.93 & $0.47-1.82$ & 0.83 & - & - & - \\
\hline 1.5- vs. 3.0-T MRI & 0.99 & $0.64-1.56$ & 0.99 & - & - & - \\
\hline Discovery LS vs. 690 & 0.77 & $0.38-1.54$ & 0.45 & - & - & - \\
\hline Stage $1-2$ vs. $3-4$ & 1.56 & $0.77-3.16$ & 0.22 & - & - & - \\
\hline $\mathrm{SUV}_{\max }>8.1(\mathrm{~g} / \mathrm{mL})$ & 1.28 & $0.64-2.54$ & 0.49 & - & - & - \\
\hline $\mathrm{SUV}_{\text {mean }}>4.5(\mathrm{~g} / \mathrm{mL})$ & 1.25 & $0.63-2.50$ & 0.52 & - & - & - \\
\hline MTV > $6.33\left(\mathrm{~cm}^{3}\right)$ & 2.63 & $1.10-6.27$ & 0.029 & 12.8 & $2.15-76.0$ & 0.005 \\
\hline TLG $>24.77\left(\mathrm{~g} \cdot \mathrm{cm}^{3} / \mathrm{mL}\right)$ & 2.27 & $0.99-5.18$ & 0.052 & - & - & - \\
\hline $\mathrm{ADC}_{\min }>939\left(10^{-6} \mathrm{~mm}^{2} / \mathrm{s}\right)$ & 0.72 & $0.32-1.63$ & 0.43 & - & - & - \\
\hline $\mathrm{ADC}_{\text {mean }}>1282\left(10^{-6} \mathrm{~mm}^{2} / \mathrm{s}\right)$ & 0.59 & $0.27-1.27$ & 0.18 & - & - & - \\
\hline DTV $>4.84\left(\mathrm{~cm}^{3}\right)$ & 2.61 & $1.06-6.44$ & 0.037 & - & - & - \\
\hline $\mathrm{MTV} / \mathrm{ADC}_{\min }$ ratio $>8.31\left(10^{3}\right)$ & 1.93 & $0.87-4.26$ & 0.10 & 0.20 & $0.04-1.0$ & 0.049 \\
\hline
\end{tabular}

$A D C$, apparent diffusion coefficient; $D S S$, disease-specific survival; $D T V$, diffusion tumour volume; $M T V$, metabolic tumour volume; $P D A C$, pancreatic ductal adenocarcinoma; $S U V$, standardised uptake value; $T L G$, total lesion glycolysis. Bonferroni-corrected $p$ value was 0.010 for MTV and 0.099 for $\mathrm{MTV} / \mathrm{ADC}_{\min }$ ratio 
Table 5 Predictive factors for PFS $(n=41)$

\begin{tabular}{|c|c|c|c|c|c|c|}
\hline \multirow[t]{2}{*}{ Variables } & \multicolumn{3}{|c|}{ Univariate analysis } & \multicolumn{3}{|c|}{ Stepwise multivariate analysis } \\
\hline & HR & $95 \% \mathrm{CI}$ & $p$ value & HR & $95 \% \mathrm{CI}$ & $p$ value \\
\hline Sex & 0.82 & $0.43-1.55$ & 0.54 & - & - & - \\
\hline 1.5- vs. 3.0-T MRI & 0.85 & $0.55-1.30$ & 0.45 & - & - & - \\
\hline Discovery LS vs. 690 & 0.62 & $0.33-1.17$ & 0.14 & - & - & - \\
\hline Stage $1-2$ vs. $3-4$ & 1.47 & $0.90-2.40$ & 0.13 & - & - & - \\
\hline $\mathrm{SUV}_{\max }>8.1(\mathrm{~g} / \mathrm{mL})$ & 1.64 & $0.84-3.20$ & 0.15 & - & - & - \\
\hline $\mathrm{SUV}_{\text {mean }}>4.49(\mathrm{~g} / \mathrm{mL})$ & 1.36 & $0.72-2.57$ & 0.35 & - & - & - \\
\hline MTV > $6.33\left(\mathrm{~cm}^{3}\right)$ & 3.11 & $1.34-7.23$ & 0.008 & - & - & - \\
\hline TLG $>14.3\left(\mathrm{~g} \cdot \mathrm{cm}^{3} / \mathrm{mL}\right)$ & 6.53 & $1.50-28.34$ & 0.012 & - & - & - \\
\hline $\mathrm{ADC}_{\min }>939\left(10^{-6} \mathrm{~mm}^{2} / \mathrm{s}\right)$ & 0.75 & $0.37-1.49$ & 0.41 & - & - & - \\
\hline $\mathrm{ADC}_{\text {mean }}>1274\left(10^{-6} \mathrm{~mm}^{2} / \mathrm{s}\right)$ & 0.88 & $0.45-1.74$ & 0.72 & - & - & - \\
\hline DTV $>4.84\left(\mathrm{~cm}^{3}\right)$ & 5.79 & $1.97-16.99$ & 0.001 & 5.79 & $1.97-17.0$ & 0.001 \\
\hline $\mathrm{MTV} / \mathrm{ADC}_{\min }$ ratio $>8.3\left(10^{3}\right)$ & 2.44 & $1.18-5.03$ & 0.016 & - & - & - \\
\hline
\end{tabular}

$A D C$, apparent diffusion coefficient; $D T V$, diffusion tumour volume; $M T V$, metabolic tumour volume; $P D A C$, pancreatic ductal adenocarcinoma; $P F S$, progression-free survival; $S U V$, standardised uptake value; $T L G$, total lesion glycolysis
We found that higher DTV predicted lower PFS, which had not previously been reported. Since DTV and MTV were collinear, this finding indicates that the tumour burden is a major prognostic biomarker.

Here, we demonstrated that MTV was a significant predicting factor for OS and DSS in operated PDAC patients. Several prior studies have also reported the prognostic value of ${ }^{18}$ F-FDG PET/CT-derived parameters [8, 9, 31, 34, 35]. However, these studies have shown high heterogeneity regarding the study population treatment (i.e., chemotherapy alone, chemoradiotherapy, surgery, or best supportive care) and the imaging protocol variables, making comparison difficult. In accordance with our present findings, Sakane et al [31] reported that SUV did not significantly predict OS. Also in agreement with our present results, two other studies [8, 9] found that MTV and TLG are independent predictors of OS and PFS in operated PDAC patients, regardless of neoadjuvant chemotherapy. Additionally, Chirindel et al [34] reported TLG as a predictive factor for PFS among unresectable PDAC patients. Interestingly, Hyun et al [35] showed that the intratumoural heterogeneity of ${ }^{18} \mathrm{~F}$-FDG uptake (i.e., entropy) was an independent predictor of OS. However, their study population pooled patients treated with either surgery or various combinations of radiochemotherapy, which may deeply influence the outcome, such that these results cannot be not compared with our study. Finally, we found a trend for $\mathrm{MTV} / \mathrm{ADC}_{\min }$ ratio as a predictor of DSS, which is in agreement with results published by Chen et al from a smaller patients' population [10]. Considering the inverse correlation between tumour grade and $\mathrm{ADC}_{\text {min }}$, this finding suggests that $\mathrm{MTV} / \mathrm{ADC}_{\text {min }}$ ratio could reflect the impact of both tumour burden and grade on patient outcome.

Our study had several limitations. To our knowledge, this is the largest reported series with untreated and resected
PDAC and with both preoperative DW-MRI and ${ }^{18} \mathrm{~F}-\mathrm{FDG}$ PET/CT imaging; however, only 61 patients could ultimately be enrolled in the radiopathological correlation analysis, despite our long inclusion period of about 10 years. Furthermore, 20 of these 61 patients were lost for follow-up. Although this could limit the analysis power, we demonstrated that several PET-MR-derived parameters had significant prognostic value regarding OS, DSS, and PFS, which had not been previously performed before. Only one reader measured PET and MRI metrics. However, we used VOI methods that were proven the most reproducible to this purpose, suggesting limited impact. Moreover, several different PET and MRI scanners were used, but PET data stability was ensured by phantom acquisition over the study period. Also, we specifically compared PET and MRI data, as well as OS, PFS, and DSS according to scanners and found no significant difference, suggesting limited impact. Finally, the tumour measurements were always performed using the most reproducible available methods for ${ }^{18} \mathrm{~F}$-FDG PET- and DWI-derived parameters, which overall suggests a limited impact on our results.

\section{Conclusions}

In patients with resectable PDAC, tumour grade correlated with $\mathrm{ADC}_{\text {min }}$ values. MTV was predictive for OS and DSS, while DTV was predictive of PFS. These findings suggest that the combination of ${ }^{18} \mathrm{~F}$-FDG PET- and DW-MRIderived parameters may be more useful for prognostic stratification of PDAC patients, compared with singlemodality imaging. 
Acknowledgements The authors would like to thank all referring physicians who kindly responded to the follow-up evaluation.

Funding information Open access funding provided by University of Lausanne. The authors state that this work has not received any funding.

\section{Compliance with ethical standards}

Guarantor The scientific guarantor of this publication is Sabine Schmidt Kobbe.

Conflict of interest The authors of this manuscript declare no relationships with any companies, whose products or services may be related to the subject matter of the article.

Statistics and biometry One of the authors (Vincent Dunet) has significant statistical expertise. No complex statistical methods were necessary for this paper.

Informed consent Written informed consent was given by the patients prior to the examinations.

Ethical approval Institutional Review Board approval was obtained.

\author{
Methodology \\ - prospective \\ - observational \\ - performed at one institution
}

Open Access This article is licensed under a Creative Commons Attribution 4.0 International License, which permits use, sharing, adaptation, distribution and reproduction in any medium or format, as long as you give appropriate credit to the original author(s) and the source, provide a link to the Creative Commons licence, and indicate if changes were made. The images or other third party material in this article are included in the article's Creative Commons licence, unless indicated otherwise in a credit line to the material. If material is not included in the article's Creative Commons licence and your intended use is not permitted by statutory regulation or exceeds the permitted use, you will need to obtain permission directly from the copyright holder. To view a copy of this licence, visit http://creativecommons.org/licenses/by/4.0/.

\section{References}

1. GBD 2017 Pancreatic Cancer Collaborators (2019) The global, regional, and national burden of pancreatic cancer and its attributable risk factors in 195 countries and territories, 1990-2017: a systematic analysis for the Global Burden of Disease Study 2017. Lancet Gastroenterol Hepatol 4:934-947

2. Vincent A, Herman J, Schulick R, Hruban RH, Goggins M (2011) Pancreatic cancer. Lancet 378:607-620

3. Raman SP, Horton KM, Fishman EK (2012) Multimodality imaging of pancreatic cancer-computed tomography, magnetic resonance imaging, and positron emission tomography. Cancer J 18: $511-522$

4. Hu SL, Yang ZY, Zhou ZR, Yu XJ, Ping B, Zhang YJ (2013) Role of SUV(max) obtained by 18F-FDG PET/CT in patients with a solitary pancreatic lesion: predicting malignant potential and proliferation. Nucl Med Commun 34:533-539
5. Yeh R, Dercle L, Garg I, Wang ZJ, Hough DM, Goenka AH (2018) The role of 18F-FDG PET/CT and PET/MRI in pancreatic ductal adenocarcinoma. Abdom Radiol (NY) 43:415-434

6. Ma W, Li N, Zhao W et al (2016) Apparent diffusion coefficient and dynamic contrast-enhanced magnetic resonance imaging in pancreatic cancer: characteristics and correlation with histopathologic parameters. J Comput Assist Tomogr 40:709-716

7. Hayano K, Miura F, Amano H et al (2013) Correlation of apparent diffusion coefficient measured by diffusion-weighted MRI and clinicopathologic features in pancreatic cancer patients. J Hepatobiliary Pancreat Sci 20:243-248

8. Im HJ, Oo S, Jung W et al (2016) Prognostic value of metabolic and volumetric parameters of preoperative FDG-PET/CT in patients with resectable pancreatic cancer. Medicine (Baltimore) 95:e3686

9. Lee JW, Kang CM, Choi HJ et al (2014) Prognostic value of metabolic tumor volume and total lesion glycolysis on preoperative (1)(8)F-FDG PET/CT in patients with pancreatic cancer. J Nucl Med 55:898-904

10. Chen BB, Tien YW, Chang MC et al (2016) PET/MRI in pancreatic and periampullary cancer: correlating diffusion-weighted imaging, MR spectroscopy and glucose metabolic activity with clinical stage and prognosis. Eur J Nucl Med Mol Imaging 43:1753-1764

11. Yamamoto T, Sugiura T, Mizuno T et al (2015) Preoperative FDGPET predicts early recurrence and a poor prognosis after resection of pancreatic adenocarcinoma. Ann Surg Oncol 22:677-684

12. Kim KS, Kwon J, Kim K, Chie EK (2017) Impact of resection margin distance on survival of pancreatic cancer: a systematic review and meta-analysis. Cancer Res Treat 49:824-833

13. Ahn SJ, Park MS, Lee JD, Kang WJ (2014) Correlation between 18F-fluorodeoxyglucose positron emission tomography and pathologic differentiation in pancreatic cancer. Ann Nucl Med 28:430 435

14. Buck AC, Schirrmeister HH, Guhlmann CA et al (2001) Ki-67 immunostaining in pancreatic cancer and chronic active pancreatitis: does in vivo FDG uptake correlate with proliferative activity? J Nucl Med 42:721-725

15. Hecht EM, Liu MZ, Prince MR et al (2017) Can diffusion-weighted imaging serve as a biomarker of fibrosis in pancreatic adenocarcinoma? J Magn Reson Imaging 46:393-402

16. Collins GS, Reitsma JB, Altman DG, Moons KG (2015) Transparent reporting of a multivariable prediction model for individual prognosis or diagnosis (TRIPOD): the TRIPOD statement. J Clin Epidemiol 68:134-143

17. Dunet V, Halkic N, Prior JO et al (2017) Detection and viability of colorectal liver metastases after neoadjuvant chemotherapy: a multiparametric PET/CT-MRI study. Clin Nucl Med 42:258-263

18. Schmidt S, Dunet V, Koehli M, Montemurro M, Meuli R, Prior JO (2013) Diffusion-weighted magnetic resonance imaging in metastatic gastrointestinal stromal tumor (GIST): a pilot study on the assessment of treatment response in comparison with 18F-FDG PET/ CT. Acta Radiol 54:837-842

19. Gnesin S, Deshayes E, Camus F, Dunet V, Prior JO, Verdun FR (2017) Quantification and monitoring of PET/CT data in multicentre trials: the Swiss SAKK 56/07 trial experience. Médecine Nucléaire 41:259-266

20. Boellaard R, Delgado-Bolton R, Oyen WJ et al (2015) FDG PET/ CT: EANM procedure guidelines for tumour imaging: version 2.0. Eur J Nucl Med Mol Imaging 42:328-354

21. Kitao T, Hirata K, Shima K et al (2016) Reproducibility and uptake time dependency of volume-based parameters on FDG-PET for lung cancer. BMC Cancer 16:576

22. Ma C, Liu L, Li J et al (2016) Apparent diffusion coefficient (ADC) measurements in pancreatic adenocarcinoma: a preliminary study of the effect of region of interest on ADC values and interobserver variability. J Magn Reson Imaging 43:407-413 
23. Brierley JD, Gospodarowicz MK, Wittekind C (2017) TNM classification of malignant tumours, 8th edn. Wiley-Blackwell

24. Kurosawa J, Tawada K, Mikata R et al (2015) Prognostic relevance of apparent diffusion coefficient obtained by diffusion-weighted MRI in pancreatic cancer. J Magn Reson Imaging 42:1532-1537

25. Ma C, Li Y, Wang L et al (2017) Intravoxel incoherent motion DWI of the pancreatic adenocarcinomas: monoexponential and biexponential apparent diffusion parameters and histopathological correlations. Cancer Imaging 17:12

26. De Robertis R, Tinazzi Martini P, Demozzi E et al (2015) Diffusion-weighted imaging of pancreatic cancer. World J Radiol 7:319-328

27. Cerny M, Dunet V, Rebecchini C et al (2019) Response of locally advanced rectal cancer (LARC) to radiochemotherapy: DW-MRI and multiparametric PET/CT in correlation with histopathology. Nuklearmedizin 58:28-38

28. Giganti F, De Cobelli F, Canevari C et al (2014) Response to chemotherapy in gastric adenocarcinoma with diffusion-weighted MRI and (18) F-FDG-PET/CT: correlation of apparent diffusion coefficient and partial volume corrected standardized uptake value with histological tumor regression grade. J Magn Reson Imaging 40: 1147-1157

29. Gu J, Khong PL, Wang S, Chan Q, Law W, Zhang J (2011) Quantitative assessment of diffusion-weighted MR imaging in patients with primary rectal cancer: correlation with FDG-PET/CT. Mol Imaging Biol 13:1020-1028

30. Schwenzer NF, Schmidt H, Gatidis S et al (2014) Measurement of apparent diffusion coefficient with simultaneous MR/positron emission tomography in patients with peritoneal carcinomatosis: comparison with 18F-FDG-PET. J Magn Reson Imaging 40: $1121-1128$

31. Sakane M, Tatsumi M, Kim T et al (2015) Correlation between apparent diffusion coefficients on diffusion-weighted MRI and standardized uptake value on FDG-PET/CT in pancreatic adenocarcinoma. Acta Radiol 56:1034-1041

32. Chen BB, Tien YW, Chang MC et al (2018) Multiparametric PET/ MR imaging biomarkers are associated with overall survival in patients with pancreatic cancer. Eur J Nucl Med Mol Imaging 45: 1205-1217

33. Garces-Descovich A, Morrison TC, Beker K, Jaramillo-Cardoso A, Moser AJ, Mortele KJ (2019) DWI of pancreatic ductal adenocarcinoma: a pilot study to estimate the correlation with metastatic disease potential and overall survival. AJR Am J Roentgenol 212: 323-331

34. Chirindel A, Alluri KC, Chaudhry MA et al (2015) Prognostic value of FDG PET/CT-derived parameters in pancreatic adenocarcinoma at initial PET/CT staging. AJR Am J Roentgenol 204: 1093-1099

35. Hyun SH, Kim HS, Choi SH et al (2016) Intratumoral heterogeneity of (18)F-FDG uptake predicts survival in patients with pancreatic ductal adenocarcinoma. Eur J Nucl Med Mol Imaging 43: $1461-1468$

Publisher's note Springer Nature remains neutral with regard to jurisdictional claims in published maps and institutional affiliations. 